\title{
Optimal time allocation in active retirement
}

\author{
Miguel Sánchez-Romero ${ }^{1,2}$ (i) $\cdot$ Alexia Prskawetz ${ }^{1,2}$ (i)
}

Published online: 25 November 2019

(C) The Author(s) 2019

\begin{abstract}
We set up a lifecycle model of a retired scholar who chooses optimally the time devoted to different activities including physical activity, continued work and social engagement. While time spent in physical activity increases life expectancy, continued scientific publications increases the knowledge stock. We show the optimal trade off between these activities in retirement and its sensitivity with respect to alternative settings of the preference parameters.
\end{abstract}

Keywords Time allocation · Active retirement $\cdot$ Longevity $\cdot$ Scientific production

\section{Motivation}

With increasing survival to old age, the share of life time spent in retirement has increased over the last decades. While many studies investigate the transition from work to retirement, the time spent in retirement is less investigated. As recently argued in Sprod et al. (2017, p. 10), "The transition from a working lifestyle to one of retirement involves a reorganization of daily activities and the choice of activities has health consequences." Hence, in light of productive and healthy aging it is important to gain a better understanding of how people spend their time in retirement.

In this paper we propose an optimal control model of productive and healthy aging in retirement. We focus our analysis on the active retirement phase of a university professor. We assume that our individual derives utility from scientific work (including research, attending conferences, etc.) and social engagement but suffers a utility

Miguel Sánchez-Romero

miguel.sanchez@econ.tuwien.ac.at

Alexia Prskawetz

afp@econ.tuwien.ac.at

1 Research Unit Economics, Institute of Statistics and Mathematical Methods in Economics, TU Wien, Wien, Austria

2 Wittgenstein Centre of Demography and Global Human Capital, Vienna Institute of Demography, Austrian Academy of Science, Vienna, Austria 
loss from physical activity. These activities in turn determine the evolution of the knowledge and health stock. We assume that a higher knowledge stock left behind is positively valued by our individual and health is positively related to survival. Hence, an optimal tradeoff between time allocated to research and physical activity will exist. We investigate the optimal time allocation in retirement dependent on various specifications of preferences and technologies of knowledge and health accumulation.

\section{The model}

We consider a retired scholar at age $x$ with a fixed but guaranteed income until death. The consumption path and the wealth profile are assumed to have been optimally chosen during the working period. Hence, we focus on the optimal allocation of the available time in retirement among the three main activities: cognitive activity $\left(t_{c}\right)$, physical activity $\left(\mathrm{t}_{h}\right)$, and social activity $\left(\mathrm{t}_{s}\right)$. Cognitive activity comprises the time devoted to producing papers, going to working meetings, attending conferences, workshops, and seminars. Physical activity accounts for the time spent on the gym, hiking in the mountains, etc; while the social activity represents the leisure time going to concerts, watching football, etc. The sum of all the available time at time $t$ is

$$
\mathrm{t}_{c}(t)+\mathrm{t}_{h}(t)+\mathrm{t}_{s}(t)=T
$$

where $T$ denotes the maximum number of available minutes per day. ${ }^{1}$ We assume that the time spent on various activities are mutually exclusive; i.e. activities cannot be conducted in parallel to each other.

The remaining length of life is assumed to be deterministic and depends on the stock of health deficits $(D)$ that in turn depends on the time spent on physical activity. To account for the evolution of health deficits we follow the work by Dalgaard and Strulik (2014). In particular, health deficits accumulate annually at a rate $\mu(D-a)$ until the maximum number of health deficits $\bar{D}$ is reached. The rate of accumulation of health deficits can be slowed down through physical activity (Dalgaard and Strulik 2017; Schuenemann et al. 2017; Strulik 2018) as follows

$$
\dot{D}=\mu\left(D-A \mathrm{t}_{h}^{\eta}-a\right)
$$

Parameter $\mu>0$ is the natural force of aging, $a>0$ reflects the exogenous rate of improvement in the accumulation of health deficits, and $A>0$ and $\eta \in(0,1)$ are parameters affecting the impact of physical activity on the accumulation of health deficits. Parameter $\eta$ is a positive constant smaller than one in order to account for the fact that the impact of physical activity on accumulation of health deficits is subject to decreasing returns to scale.

To represent the daily activity of a scientist, we assume our scholar is interested in working with doctoral students and colleagues in order to contribute to the stock of knowledge, which is denoted by $K$. The stock of knowledge at age $t$ is defined as the

\footnotetext{
1 The value of $T$ denotes the total minutes per day net of sleep and other leisure activities.
} 
total number of papers written until age $t, K(t)$. To produce papers, the scholar needs to devote time to cognitive activity. The number of papers published at each time is positively related to the exiting stock of knowledge of our scholar. Thus, the number of papers published at time $t$ is given $b^{2}$

$$
\dot{K}=\theta \mathrm{t}_{c}^{\gamma} K^{\beta},
$$

where $\gamma \in(0,1)$ and $\beta \in(0,1)$ are, respectively, the returns of the time devoted to cognitive activities and of knowledge, and $\theta>0$ is a constant parameter representing the overall productivity of the scholar. Solving (3) recursively yields that the stock of knowledge at time $t$ is:

$$
K(t)=\left(K(x)^{1-\beta}+\int_{x}^{t}(1-\beta) \theta\left(\mathrm{t}_{c}(s)\right)^{\gamma} d s\right)^{\frac{1}{1-\beta}} .
$$

Moreover our scientist is aware that the stock of knowledge will improve the wellbeing of future generations. Thus, the stock of knowledge left to future generations, $K(\alpha)$, is assumed to provide utility to the scholar as captured by a salvage-value function $S(K(\alpha))=\kappa K(\alpha)$, similar to Feichtinger et al. (2019).

The lifetime utility is given by

$$
\int_{x}^{\alpha} e^{-\rho(t-x)} U\left(\mathrm{t}_{s}, \mathrm{t}_{c}, \mathrm{t}_{h}\right) d t+e^{-\rho(\alpha-x)} S(K(\alpha))
$$

where $\rho$ is the subjective discount factor and $U\left(\mathrm{t}_{s}, \mathrm{t}_{c}, \mathrm{t}_{h}\right)$ is the instantaneous utility from the different activities. For convenience, we assume the following functional form for the instantaneous utility:

$$
U\left(\mathrm{t}_{s}, \mathrm{t}_{c}, \mathrm{t}_{h}\right)=\phi_{s} \log \mathrm{t}_{s}+\phi_{c} \log \mathrm{t}_{c}-\phi_{h} \frac{\mathrm{t}_{h}^{1+\omega}}{1+\omega},
$$

where parameters $\phi_{s}, \phi_{c}, \phi_{h}$ are the weights of each instantaneous (dis)utility from each activity on $U$. We use logarithmic utility functions on $t_{s}$ and $t_{c}$ and a convex function on the disutility of health activities in order to guarantee the existence and uniqueness of an optimal solution.

\section{Optimal solution}

Our retired scholar chooses the time devoted to cognitive activities, $\mathrm{t}_{c}$, physical activities, $\mathrm{t}_{h}$, and social activities, $\mathrm{t}_{s}$, that maximize the lifetime utility function (5) subject to the time constraint (1), the dynamics of accumulation of health deficits (2), the dynamics of the accumulation of knowledge (3), the initial stock of knowledge, $K(x)=K_{x}$,

\footnotetext{
2 We follow the scientific production used by Feichtinger et al. (2019) and assume for simplicity no reputation $(R=0)$ and $\alpha=1$. These two assumptions imply that knowledge is equivalent to the number of papers $(P=K)$ as modeled in Feichtinger et al. (2019).
} 
the initial stock of health deficits, $D(x)=D_{x}$, and the final boundary condition $D(\alpha)=\bar{D}$. In "Appendix A" we derive the necessary conditions for this problem using the calculus of variations (Portryagin's Maximum Principle). We solve the following optimal control problem:

$$
\max _{\mathrm{t}_{s}(\cdot), \mathrm{t}_{c}(\cdot), \mathrm{t}_{h}(\cdot)} \int_{x}^{\alpha} e^{-\rho(t-x)} U\left(\mathrm{t}_{S}(t), \mathrm{t}_{c}(t), \mathrm{t}_{h}(t)\right) d t+e^{-\rho(\alpha-x)} S(K(\alpha))
$$

$$
\begin{gathered}
\text { s.t. } \dot{D}(t)=\mu\left(D(t)-A \mathrm{t}_{h}(t)^{\eta}-a\right) \\
\dot{K}(t)=\theta \mathrm{t}_{c}(t)^{\gamma} K(t)^{\beta} \\
T=\mathrm{t}_{s}(t)+\mathrm{t}_{c}(t)+\mathrm{t}_{h}(t), \\
K(x)=K_{x}, \\
D(x)=D_{x}, \\
D(\alpha) \leq \bar{D} .
\end{gathered}
$$

The current value Hamiltonian associated to (7) is

$$
\mathcal{H}=U\left(\mathrm{t}_{s}, \mathrm{t}_{c}, \mathrm{t}_{h}\right)+\lambda_{K} \theta \mathrm{t}_{c}^{\gamma} K^{\beta}+\lambda_{D} \mu\left(D-A \mathrm{t}_{h}^{\eta}-a\right),
$$

where $\lambda_{K}$ and $\lambda_{D}$ are the "adjoint" or "co-state" variables associated to knowledge and health deficits, respectively. In economic terms, $\lambda_{K}$ reflects the value given by our scholar to produce one additional paper, while $\left(-\lambda_{D}\right)$ is the value given by our scholar to avoid the accumulation of health deficits. Thus, $\left(-\lambda_{D}\right)$ is a measure of the willingness to pay for saving a life.

First-order conditions (FOCs) Using (6), differentiating (8) with respect to $t_{c}$ and $t_{h}$ and equating the results to zero yields:

$$
\begin{array}{r}
\operatorname{MUC}_{\mathrm{t}_{c}}(t)=\frac{\phi_{s}}{T-\mathrm{t}_{c}-\mathrm{t}_{h}}-\frac{\phi_{c}}{\mathrm{t}_{c}}=\lambda_{K} \gamma \theta \mathrm{t}_{c}{ }^{\gamma-1} K^{\beta}, \\
\mathrm{MUC}_{\mathrm{t}_{h}}(t)=\frac{\phi_{s}}{T-\mathrm{t}_{c}-\mathrm{t}_{h}}+\phi_{h} \mathrm{t}_{h}{ }^{\omega}=\left(-\lambda_{D}\right) \eta \mu A \mathrm{t}_{h}{ }^{\eta-1} .
\end{array}
$$

Condition (9) states that the optimal $\mathrm{t}_{c}(t)$ satisfies that the marginal utility cost of cognitive activities, i.e. $\mathrm{MUC}_{\mathrm{t}_{c}}(t)$, must be equal to the marginal value of the time 
devoted to cognitive activities. The marginal utility cost of cognitive activities is the difference between the marginal utility lost by not spending time on social activities, $\phi_{s} / \mathrm{t}_{s}$, and the marginal utility gain from the time devoted to cognitive activities, $\phi_{c} / \mathrm{t}_{c}$, which from (9) must be positive; i.e. $\frac{\phi_{s}}{t_{s}}-\frac{\phi_{c}}{t_{c}}>0$. The marginal value of cognitive activities is the additional paper produced [right-hand side of Eq. (9)]. Condition (10) implies that the optimal time devoted to physical activities satisfies that the marginal utility cost of health activities, $\mathrm{MUC}_{\mathrm{t}_{h}}(t)$, is equal to the marginal value of the time devoted to health activities. The marginal utility cost of health activities is given by the sum of the marginal utility lost by not spending time on social activities plus the marginal (dis)utility from physical activities. The marginal value of time devoted to health activities is the reduction in the accumulation of health deficits.

Envelope conditions and transversality conditions From (7) we have two transversality conditions (TCs) and two envelope conditions (ECs). The TC associated to the accumulation of health deficits is

$$
\lambda_{D}(\alpha)=-v \text { with } v>0
$$

where $v$ is the Kuhn-Tucker multiplier that results from the constraint on the maximum number of health deficits; i.e. $D(\alpha)=\bar{D}$. The EC associated to the stock of health deficits is

$$
\frac{\dot{\lambda}_{D}}{\lambda_{D}}=\rho-\mu .
$$

According to (12) impatience, which is reflected by $\rho$, drives the price of avoiding the accumulation of health deficits up, while the natural rate of aging, $\mu$, reduces the willingness to pay for avoiding the accumulation of health deficits. Solving (12) yields

$$
-\lambda_{D}(t)=-\lambda_{D}(\alpha) e^{(\rho-\mu)(t-\alpha)} .
$$

Thus, Eq. (13) shows that when $\mu>\rho$ our scholar is less willing to pay for avoiding the accumulation of health deficits as time passes by, since the investment becomes less efficient with age.

Assuming $S(K(\alpha))=\kappa K(\alpha)$, which is similar to that used by Feichtinger et al. (2019), the transversality condition (TC) for the accumulation of knowledge is ${ }^{3}$

$$
\lambda_{K}(\alpha)=\kappa
$$

where $\kappa$ represents the marginal value of knowledge at the end of life. The envelope condition (EC) for the accumulation of knowledge is

$$
\frac{\dot{\lambda}_{K}}{\lambda_{K}}=\rho-\beta \frac{\dot{K}}{K} .
$$

\footnotetext{
3 Feichtinger et al. (2019) assume that the salvage-value function of a scientist, who cares both about the stock of knowledge and about reputation, is $\kappa_{1} K(\alpha)+\kappa_{2} R(\alpha)$.
} 
Thus, (15) suggests that the marginal value of knowledge first decreases with age when $\beta \frac{\dot{K}}{K}>\rho$, since the stock of knowledge is initially small, and once the stock of knowledge is sufficiently large and $\rho>\beta \frac{\dot{K}}{K}$ holds, it increases with age, ceteris paribus the behavior of the scientist over the lifecycle.

Terminal age condition Our scholar chooses a terminal age, $\alpha^{*}$, that satisfies

$$
\mathcal{H}\left(\alpha^{*}\right)+\left.\frac{d S(K(\alpha))}{d \alpha}\right|_{\alpha=\alpha^{*}}=\rho S\left(K\left(\alpha^{*}\right)\right)
$$

and

$$
D\left(\alpha^{*}\right)=\bar{D}
$$

Condition (16) shows that the remaining lifetime utility is maximized when the marginal utility of living one additional instant (left-hand side) equals the marginal utility loss of not enjoying the "glory" from the stock of knowledge left to future generations (right-hand side). Solving (16) we obtain the shadow price of avoiding the accumulation of health deficits at age $\alpha^{*}$

$$
-\lambda_{D}\left(\alpha^{*}\right)=\frac{\mathrm{U}\left(\alpha^{*}\right)+2 \mathrm{t}_{c}\left(\alpha^{*}\right) \frac{\mathrm{MUC}_{\mathrm{t}_{c}}\left(\alpha^{*}\right)}{\gamma}+\mathrm{t}_{h}\left(\alpha^{*}\right) \frac{\mathrm{MUC}_{\mathrm{t}_{h}}\left(\alpha^{*}\right)}{\eta}-\rho \kappa K^{*}\left(\alpha^{*}\right)}{\mu(\bar{D}-a)} .
$$

Equation (18) shows that the shadow price of health deficits at age $\alpha^{*},-\lambda_{D}\left(\alpha^{*}\right)$, is positively related to the utility at the end of life, $\mathrm{U}\left(\alpha^{*}\right) \equiv U\left(\mathrm{t}_{s}\left(\alpha^{*}\right), \mathrm{t}_{c}\left(\alpha^{*}\right), \mathrm{t}_{h}\left(\alpha^{*}\right)\right)$, the value from producing more knowledge, $2 \mathrm{t}_{c}\left(\alpha^{*}\right) \mathrm{MUC}_{\mathrm{t}_{c}}\left(\alpha^{*}\right) / \gamma$, and the value of avoiding the accumulation of health deficits, $\mathrm{t}_{h}\left(\alpha^{*}\right) \mathrm{MUC}_{\mathrm{t}_{h}}\left(\alpha^{*}\right) / \eta$. Whereas the shadow price of health deficits at age $\alpha^{*}$ is negatively related to the marginal utility loss of not enjoying the "glory" from the stock of knowledge left to future generations, $\rho \kappa K^{*}\left(\alpha^{*}\right)$. Consequently, the term $\rho \kappa K^{*}\left(\alpha^{*}\right)$ indicates that impatient scholars, who have many papers published, are less willing to pay for avoiding the accumulation of health deficits than more patient scholars.

\section{Calibration and simulation strategy}

We use our model to study what kind of preferences allow scholars to enjoy a long retirement life, while continuing to accumulate scientific knowledge.

\subsection{Calibration}

We calibrate the model to the scientific life of Prof. Dr. Gustav Feichtinger. Our simulation results are based on the following three facts. First, our professor goes to the gym $3 \mathrm{~h}$ per week and he also likes hiking on weekends. Second, our professor has produced 378 papers up to age $78\left(K_{x}\right)$ with an average publication rate of 7 papers 


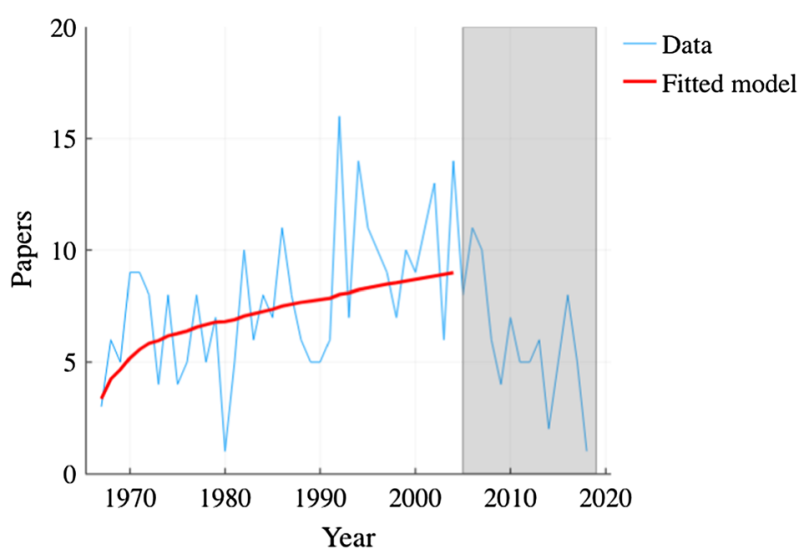

Fig. 1 Production of papers by Prof. Dr. Gustav Feichtinger. Note: The shaded area depicts the retirement period of Prof. Dr. Gustav Feichtinger

per year (see Fig. 1). After retirement, our professor has published on average around 5 articles per year (see the gray area in Fig. 1). The red solid line shows the fit of Eq. (3) to the scientific production of our professor, which gives an estimate for $\beta$ of 0.21 with a standard error of 0.07 . The overall scientific productivity, $\theta=0.01$, and the returns to cognitive time, $\gamma=0.7$, are calibrated to obtain an average number of papers published per year of 5. Third, based on cohort deaths rates of Austrian males born in 1940 from the Human Mortality Database (2018), we obtain that the average life expectancy at age 78 is 13 years. However, given that academicians enjoy a longevity advantage of 7 years relative to the general population (Winkler-Dworak 2008; Winkler-Dworak and Kaden 2014), we calibrate Eq. (2) so as to give an average longevity of 98.5 years. Specifically, we follow Mitnitski et al. (2002a, b) and set the value of the natural rate of ageing $(\mu)$ and the exogenous rate of improvements in the accumulation of health deficits $(a)$ at 0.04 and 0.02 , respectively. The initial stock of health deficits $D_{x}$ is set at 0.106 , which corresponds to the estimated value of health deficits at age 78 reported by Mitnitski et al. (2002a), and the maximum number of health deficits $\bar{D}$ at 0.22 . We chose the value of $\bar{D}$ that guarantees our scholar to reach 97.5 years without devoting time to physical activities. Then, we calibrate the parameters $A$ and $\eta$ that account for the impact of physical activity on the accumulation of health deficits to 0.000363 and 0.7 , respectively. The values of $A$ and $\eta$ are set so as to obtain a difference between the highest longevity $-720 \mathrm{~min}$ per day devoted to health activities - and the lowest longevity - 0 min on health activities - of 7.35 years, which corresponds to the standard deviation of the life expectancy at age 78 for an Austrian male born in 1940 (Human Mortality Database 2018).

Table 1 shows all the parameter values used in the model. For the sake of convenience, and without loss of generality, we have fixed the utility weight of physical activity $\left(\phi_{h}\right)$ to 0.015 and the elasticity of physical activity $(\omega)$ to 0.1 . The value of $\phi_{h}$ is much lower than the assumed values for $\phi_{s}$ and $\phi_{c}$ in order to have comparable instantaneous utility values between social and cognitive activities, which are in logs, 
Table 1 Parameter values

\begin{tabular}{|c|c|c|c|c|c|}
\hline Parameter & Symbol & Value & Parameter & Symbol & Value \\
\hline Time & & & Knowledge & & \\
\hline Max. time per day (min) & $\mathrm{T}$ & 720 & Initial stock & $K_{x}$ & 378 \\
\hline \multirow[t]{2}{*}{ Current age } & $x$ & 78 & Returns to knowledge & & \\
\hline & & & & $\theta$ & 0.0126 \\
\hline Preferences & & & & $\gamma$ & 0.7 \\
\hline Fixed & & & & $\beta$ & 0.214819 \\
\hline Elasticity physical activity & $\omega$ & 0.1 & Health deficits & & \\
\hline Weight physical act. & $\phi_{h}$ & 0.015 & Natural force of aging & $\mu$ & 0.043 \\
\hline Not fixed & & & & $a$ & 0.02 \\
\hline Weight cognitive activity & $\phi_{c}$ & $(2,20)$ & Impact of physical activity & $A$ & 0.000363 \\
\hline Weight social activity & $\phi_{s}$ & $\left(\phi_{c}, 20\right)$ & & $\eta$ & 0.7 \\
\hline Subj. discount factor & $\rho$ & $(0,0.02)$ & Health deficits at $x$ & $D_{x}$ & 0.106 \\
\hline Salvage parameter & $\kappa$ & $(0,5)$ & Maximum deficits & $\bar{D}$ & 0.22 \\
\hline
\end{tabular}

and physical activity that is represented by a positive and strictly convex function. ${ }^{4}$ Given that we do not know the preference of our scholar towards the different activities, we examine alternative values for all the remaining parameters of the utility function. Specifically, we consider that the utility weight of cognitive activities $\phi_{c}$ may range between 2 and 20, and the utility weight on social activities $\phi_{s}$ between $\phi_{c}$ and 20. We consider $\phi_{s}$ to be always equal or greater than $\phi_{c}$ in order to guarantee that the first-order condition for an optimal time devoted to cognitive activities is satisfied; i.e. $\phi_{s} / \mathrm{t}_{s}-\phi_{c} / \mathrm{t}_{c}>0$. The range of values for $\phi_{s}$ and $\phi_{c}$ are chosen in order to account for the impact of different weights of physical activity on the utility function. In particular, given that $\phi_{h}$ and $\omega$ are fixed, an increase in the values of $\phi_{c}$ and $\phi_{s}$ implies a decline in the relative importance of the disutility of physical activity on the instantaneous utility. The subjective discount factor is considered to take values between 0 and 0.02 , which includes the average discount factor of 0.01 , and the salvage parameter ranges between 0 and 5 .

\subsection{Simulation strategy}

To account for all possible preferences, we assume the set of preference weights $\psi=\left\{\kappa, \rho, \phi_{c}, \phi_{s}\right\}$ to be unknown and described by the uninformative prior-i.e., uniform distribution-density $q(\psi)$. Then, we use our deterministic model $M$, that maps $\psi \in \Psi \subseteq \mathbb{R}_{+}^{4}$ into a set of output variables $\pi \in \Pi \subseteq \mathbb{R}_{+}^{2}$ :

$$
M: \Psi \rightarrow \Pi
$$

\footnotetext{
4 Indeed, it can be shown that for $\phi_{c}=\phi_{S}=1$ and if our scholar splits his time equally among the three different activities, the disutility from physical activity will be equal to the utility gained from social or cognitive activities; i.e. $0.015 \frac{(T / 3)^{1+0.1}}{1+0.1}=\log (T / 3)$.
} 


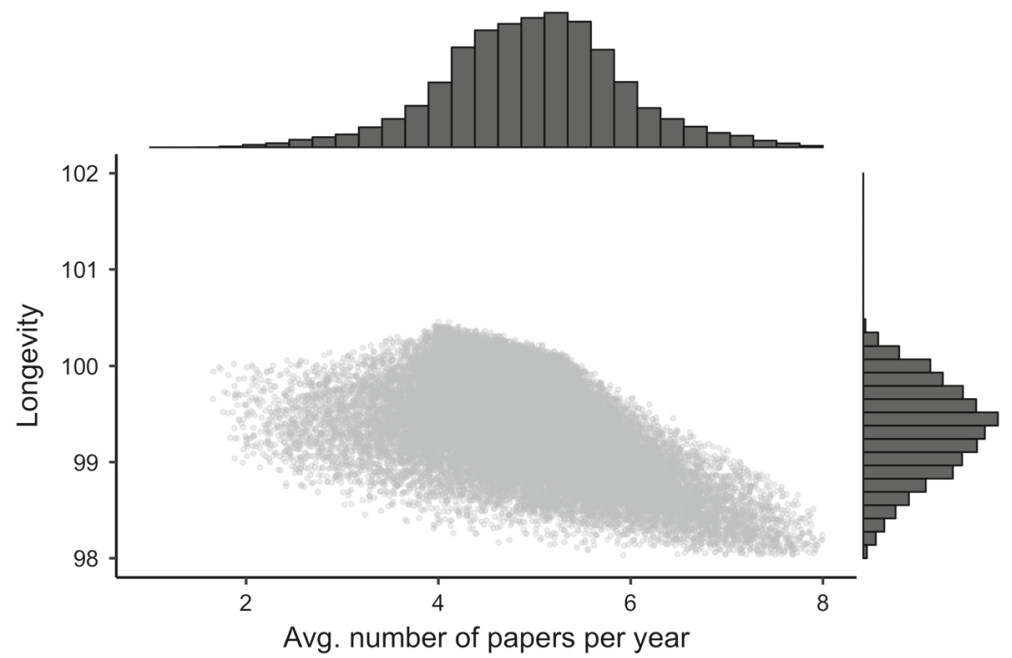

Fig. 2 Set of possible longevity $(\alpha)$ and average number of papers $(p)$ generated with the benchmark model

to generate output densities $f(\pi)$. In particular, we run 33,000 random draws from the priors and generate densities for the two outputs: longevity $(\alpha)$ and average number of papers per year during retirement $(p)$, which is calculated as $(K(\alpha)-378) /(\alpha-78)$, where 378 is the total number of papers published by our scholar until age 78 .

Figure 2 shows the combination of possible average number of papers published per year $(p)$ and longevity $(\alpha)$, i.e. $f(\alpha, p)$, for all possible sets of preference weights $\Psi$. Each gray dot in Fig. 2 corresponds to a set of possible $\psi$ values drawn from $q(\psi)$. The shape of the joint density $f(\alpha, p)$ is due to the fact that not all combinations of longevity and number of papers per year are possible given the model $M$, the set of preference weights, and the boundary conditions. The marginal densities of $p$ and $\alpha$ are also shown at the top margin and at the right margin of Fig. 2, respectively.

According to (7) our scholar must devote time to each activity in order to attain a specific combination of average number of papers per year and longevity values. The average time devoted to each activity over the remaining lifespan, given the set of possible preference weights $\Psi$, is shown in Fig. 3. Blue dots depict the average time devoted to cognitive activities, green dots depict the average time devoted to social activities, and red dots correspond to the average time devoted to physical activities over the remaining life. Solid lines represent the mean of the average time devoted to each activity conditional on the maximum longevity (left panel) and average number of papers per year (right panel). From Fig. 3 we can observe that there is almost a one-to-one relationship between the physical activity and longevity (see left panel) and between cognitive activity and the average number of papers published per year (see right panel). The lack of a cloud of points around the mean of the average time devoted to physical activity implies that the maximum longevity is determined by $t_{h}$ (see left panel), while the average number of papers per year is determined by the average time devoted to cognitive activity (see right panel). However, cognitive and social activities do not necessarily lead to a longer longevity, as the cloud of points 

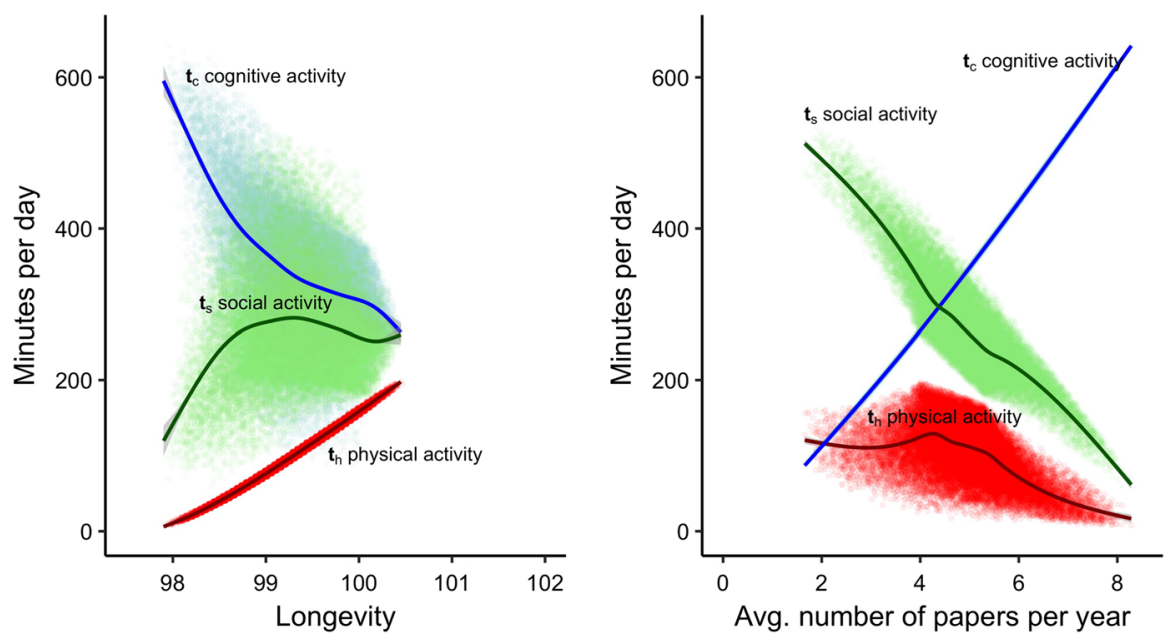

Fig. 3 Average time devoted to each activity over the remaining life by longevity (left panel) and average number of papers per year (right panel): benchmark

in the left panel suggests, as well as social and physical activities are not directly translated, according to the cloud of points in the right panel, into a higher number of papers published per year. As a consequence, for instance, Fig. 3 shows that our scholar would need to devote around $5.8 \mathrm{~h}(=350 \mathrm{~min})$ per day on cognitive activity in order to produce over the remaining lifespan an average of five papers per year (see right panel), while whenever our scholar does not target a particular longevity the time spent on social activities and on physical activities is more flexible. By contrast, given that the total available time is assumed to be fixed, when our scholar seeks a specific average number of papers per year and a specific longevity, then the average time devoted to each activity is almost completely determined. Thus, we will use this information to study the optimal distribution of time across the different activities based on the outputs.

\section{Results}

Based on the pooled sample of 33,000 random draws of preference parameters as in Fig. 2, we study the behavioral characteristics that represent our scholar. We plot three rectangles in Fig. 4. Rectangle A indicates the most likely combination (in terms of the three stylized facts described at the beginning of Sect. 4.1) of the average number of papers published per year, over the remaining life, and the longevity of our scholar, which we consider from now on our benchmark. Rectangle B represents the combination of preference weights that make our scholar to publish the same average number of papers as in A but reach an average longevity of 100 years. Rectangle C depicts the combination of preference weights that allow our scholar to live up to 100 years although at the expense of publishing an average of four papers per year. 


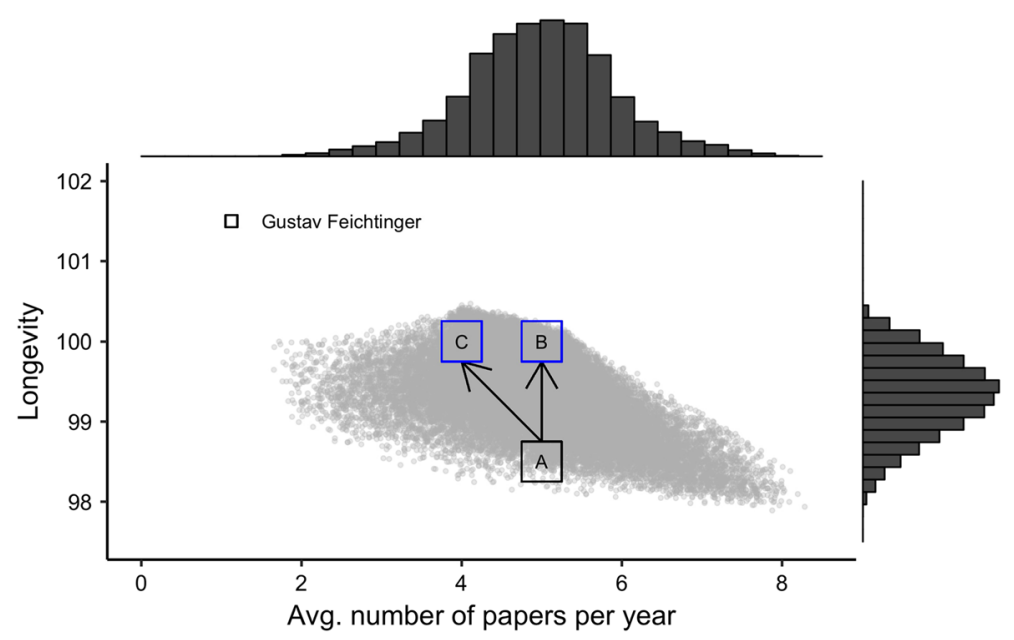

Fig. 4 Set of possible longevity $(\alpha)$ and average number of papers $(p)$ generated with the benchmark model

Table 2 Descriptive statistics of the preference parameters under three different scenarios

\begin{tabular}{|c|c|c|c|c|c|c|c|}
\hline & \multirow[t]{2}{*}{ Symbol } & \multicolumn{2}{|c|}{ Benchmark (A) } & \multicolumn{2}{|l|}{ Case B } & \multicolumn{2}{|l|}{ Case C } \\
\hline & & Mean & $\mathrm{SD}$ & Mean & $\mathrm{SD}$ & Mean & SD \\
\hline Avg. number of papers per year & $p$ & 5.02 & 0.14 & 4.99 & 0.14 & 4.06 & 0.14 \\
\hline Longevity & $\alpha$ & 98.61 & 0.11 & 99.94 & 0.11 & 100.01 & 0.14 \\
\hline Weight cognitive activity & $\phi_{c}$ & 5.078 & 1.389 & 16.702 & 2.489 & 14.663 & 2.971 \\
\hline Weight social activity & $\phi_{s}$ & 12.662 & 3.920 & 18.537 & 1.446 & 18.233 & 1.507 \\
\hline Subjective discount factor & $\rho$ & 0.016 & 0.004 & 0.003 & 0.002 & 0.005 & 0.003 \\
\hline Salvage parameter & $\kappa$ & 2.512 & 1.345 & 3.182 & 0.707 & 0.727 & 0.646 \\
\hline
\end{tabular}

Table 2 shows the descriptive statistics of the preference weights within each rectangle. In rectangle A we obtain that publishing around five papers per year and living slightly above 98.5 years is associated to scholars with a high preference for social activities relative to cognitive and physical activities, with a high discount factor (impatient), and with a high marginal value of knowledge. Living longer but publishing the same number of papers as in $\mathrm{A}$ - i.e rectangle $\mathrm{B}$ - is associated to scholars with a high marginal value of the stock of knowledge left to future generations, with a low discount factor, and with a low value of the disutility of physical activities. By contrast, publishing less papers but living longer, as in rectangle $\mathrm{C}$, is achieved by scholars who give a low marginal value to the stock of knowledge left to future generations, have a low time discount factor, and give a low value to the disutility of physical activity.

Table 3 reports the average distribution of time in each scenario. In the benchmark scenario (A) we obtain that in order to be able to publish five papers per year and reach a longevity of 98.6 years, our scholar has to devote on average $49 \%(=350.4 / 720)$ of the available time to cognitive activities, $45 \%$ to social activities, and $7 \%$ of the time to physical activities. To increase the longevity by one year, while keeping the scientific 
Table 3 Average distribution of time over the remaining life under three different scenarios

\begin{tabular}{|c|c|c|c|c|c|c|c|c|c|c|}
\hline & \multirow[t]{2}{*}{ Symbol } & \multicolumn{3}{|c|}{ Benchmark (A) } & \multicolumn{3}{|c|}{ Case B } & \multicolumn{3}{|c|}{ Case C } \\
\hline & & Mean & & $\mathrm{SD}$ & Mean & & SD & Mean & & $\mathrm{SD}$ \\
\hline Avg. number of papers per year & $p$ & 5.0 & & 0.14 & 5.0 & & 0.14 & 4.1 & & 0.14 \\
\hline Longevity & $\alpha$ & 98.6 & & 0.11 & 99.9 & & 0.11 & 100.0 & & 0.14 \\
\hline \multicolumn{2}{|c|}{ Distribution of time over remaining life: } & \multicolumn{3}{|c|}{ in $\%$} & \multicolumn{3}{|c|}{ in $\%$} & \multicolumn{3}{|c|}{ in $\%$} \\
\hline Cognitive activity in min/day & $\mathrm{t}_{c}$ & 350 & 49 & 11.5 & 348 & 48 & 12.1 & 270 & 37 & 11.1 \\
\hline Social activity in min/day & $\mathrm{t}_{s}$ & 322 & 45 & 13.5 & 219 & 30 & 14.2 & 291 & 40 & 16.9 \\
\hline Physical activity in min/day & $\mathrm{t}_{h}$ & 48 & 7 & 7.4 & 153 & 21 & 9.6 & 160 & 22 & 12.0 \\
\hline
\end{tabular}

production unchanged, as indicated in case $\mathrm{B}$, our scholar should reduce the time on social activities from 45 to $30 \%$ and increase the time devoted to physical activities from 7 to $21 \%$. However, in order to live half a year longer than in case B (see case C), our scholar needs to reduce the scientific production from 48 to $37 \%$ and increase the physical activity from 21 to $22 \%$.

\section{Extensions}

In this section we propose three extensions to the optimal time allocation model (7). First, we assume the time devoted to physical activities does not generate disutility, i.e. $\phi_{h}=0$. This model represents well the behavior of a "sportsman". Second, we consider that the utility from the different activities declines as the stock of health deficits rises. We name this model as "health dependent utility" model. Third, we assume that our scholar's productivity declines with increasing health deficits. This last model is named "cognitive decay" model.

\subsection{Sportsman}

In the first extension we assume that the utility of our scholar is not reduced by devoting time to physical activities. Thus, we set $\phi_{h}=0$ so that the utility depends only on the time devoted to social and cognitive activities

$$
U\left(\mathrm{t}_{s}, \mathrm{t}_{c}, \mathrm{t}_{h}\right)=\phi_{s} \log \mathrm{t}_{s}+\phi_{c} \log \mathrm{t}_{c} .
$$

Figure 5 shows the set of possible values for longevity and average number of papers under the benchmark model (red circles) and under the sportsman model (blue triangles). By comparing the marginal distributions generated by both models, we can observe that a scholar who behaves as a sportsman will live on average longer (see the right margin) at the expense of publishing on average less papers per year (see the top margin).

The upward shift in longevity and the reduction in the number of papers in the sportsman model, shown in Fig. 5, are achieved by changing the optimal distribution 


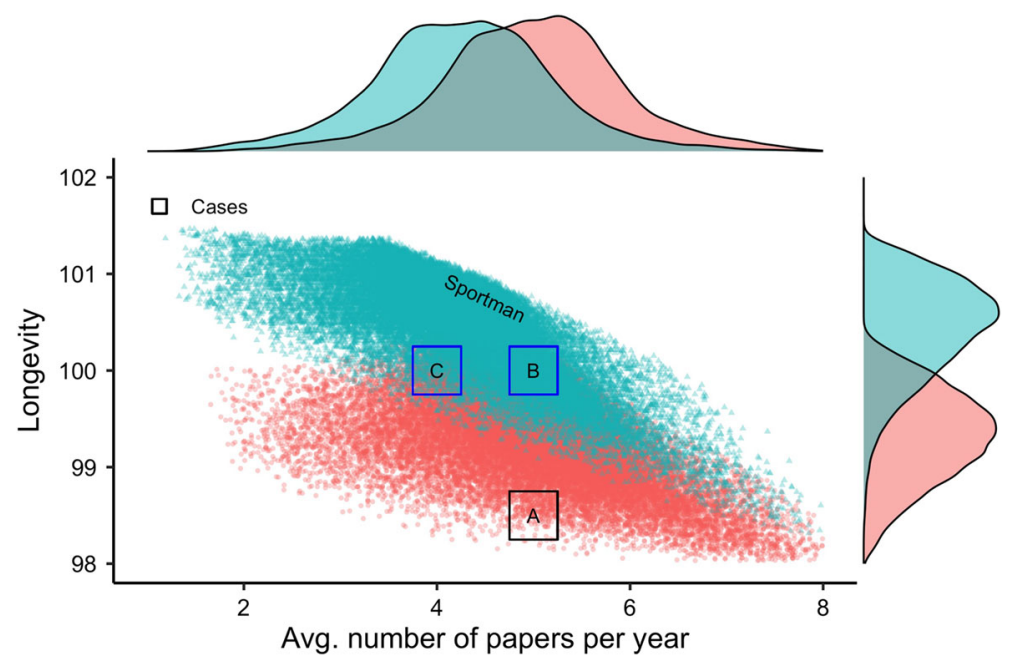

Fig. 5 Set of possible longevity $(\alpha)$ and average number of papers $(p)$ generated with the benchmark model (red circles) and the sportsman model (blue triangles) (color figure online)
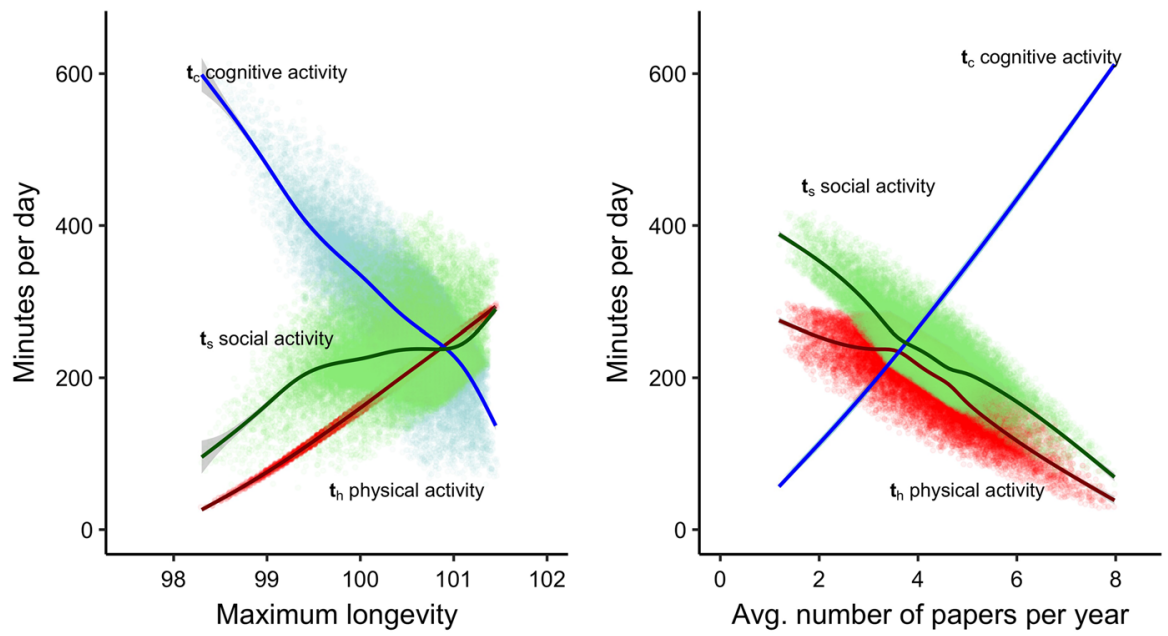

Fig. 6 Average time devoted to each activity over the remaining life by longevity (left panel) and average number of papers per year (right panel): sportsman model. Note: Solid lines represent the conditional mean over the average time devoted to each activity

of time. Figure 6 shows the optimal average time devoted to each activity over the remaining life under the sportsman model. As it can be seen in Fig. 6 this alternative model leads our scholar to devote more time to prevent the accumulation of health deficits and, as a consequence, longevity rises at the expense of reducing the average number of papers published.

Table 4 shows the descriptive statistics of the new preference weights characterizing cases A, B, and C in Fig. 5. The first thing to notice is that under the "sportsman" 
Table 4 Descriptive statistics of the preference parameters for cases A, B, and C

\begin{tabular}{|c|c|c|c|c|c|c|c|}
\hline & \multirow[t]{2}{*}{ Symbol } & \multicolumn{2}{|c|}{ Case A } & \multicolumn{2}{|l|}{ Case B } & \multicolumn{2}{|l|}{ Case C } \\
\hline & & Mean & SD & Mean & $\mathrm{SD}$ & Mean & $\mathrm{SD}$ \\
\hline Avg. number of papers per year & $p$ & - & - & 4.99 & 0.14 & 4.04 & 0.14 \\
\hline Longevity & $\alpha$ & - & - & 100.03 & 0.14 & 100.10 & 0.13 \\
\hline Weight cognitive activity & $\phi_{c}$ & - & - & 13.471 & 4.432 & 8.457 & 3.297 \\
\hline Weight social activity & $\phi_{s}$ & - & - & 16.177 & 3.320 & 16.079 & 2.976 \\
\hline Subjective discount factor & $\rho$ & - & - & 0.013 & 0.004 & 0.016 & 0.003 \\
\hline Salvage parameter & $\kappa$ & - & - & 3.687 & 0.794 & 2.434 & 0.882 \\
\hline
\end{tabular}

model only cases B and C, which both give an average longevity of 100 years, are possible. Second, comparing cases $\mathrm{B}$ and $\mathrm{C}$ we observe that publishing one less paper per year-from 5 papers to 4 -is associated to scholars who get lower utility from cognitive activities, have a lower marginal value of knowledge, and discount the future pay-offs more.

\subsection{Health dependent utility}

In the second extension we assume decreasing pay-offs with increasing health deficits. This is equivalent to assume that our scholar enjoys social activities and cognitive activities less as the number of health deficits rises. This can be implemented by multiplying the instantaneous utility function with the ratio $(\mathcal{D} / D)^{\varepsilon}$. Thus, the instantaneous utility becomes

$$
U\left(D, \mathrm{t}_{s}, \mathrm{t}_{c}, \mathrm{t}_{h}\right)=\left(\frac{\mathcal{D}}{D}\right)^{\varepsilon}\left(\phi_{s} \log \mathrm{t}_{s}+\phi_{c} \log \mathrm{t}_{c}\right)-\phi_{h} \frac{\mathrm{t}_{h}^{1+\omega}}{1+\omega}
$$

where $\mathcal{D} \in(0, D)$ is an arbitrary constant value of the stock of health deficits and $\varepsilon>0$ accounts for the rate of decline in utility caused by increases in health deficits. The constant term $\mathcal{D}>0$ is the measure with which individuals compare their health deficits (Strulik 2019). We set $\mathcal{D}$ to the health deficits at age 78 ; i.e. $\mathcal{D}=D_{x}$. To understand to what extent this new model can modify the optimal time allocation across the different activities, we assume $\varepsilon=0.5$. The value of $\varepsilon$ is ten times higher than the value assumed by Schuenemann et al. (2017) and Strulik (2019). As a result, our calibrated $\varepsilon$ implies that our scholar would value his utility from social and cognitive activities twenty five percent less at the end of life compared to his utility at age $78 .^{5}$

Figure 7 shows that this alternative model (labeled as "health dependent utility") gives slightly lower results for the longevity and slightly higher results for the average number of papers published compared to our benchmark model. This is because the relative disutility of health increases as the pay-off from social and cognitive activities declines. Hence, the individual devotes less time to physical activity. Moreover,

\footnotetext{
${ }^{5}$ Given that $D(\alpha)=\bar{D}$ and $\mathcal{D}=D_{x}$ we have that $(\mathcal{D} / D(\alpha))^{\varepsilon}=(0.106 / 0.22)^{0.5} \approx 0.75$, which is approximately $25 \%$ lower than the ratio $(\mathcal{D} / D(x))^{\varepsilon}$ at age $x$.
} 


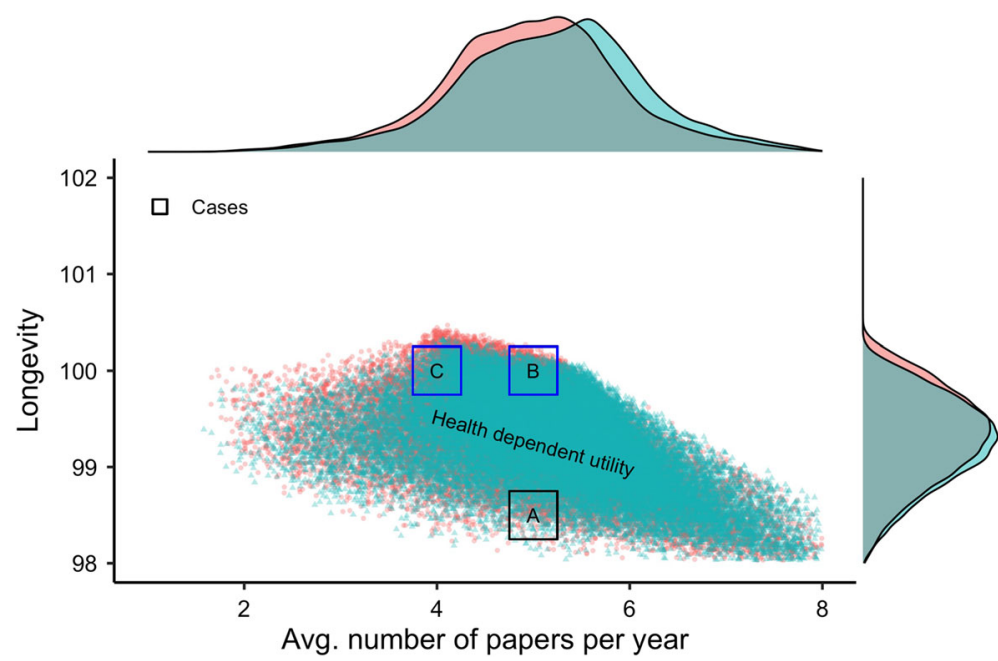

Fig. 7 Set of possible longevity $(\alpha)$ and average number of papers ( $p$ ) generated with the benchmark model (red circles) and the health dependent utility model (blue triangles) (color figure online)
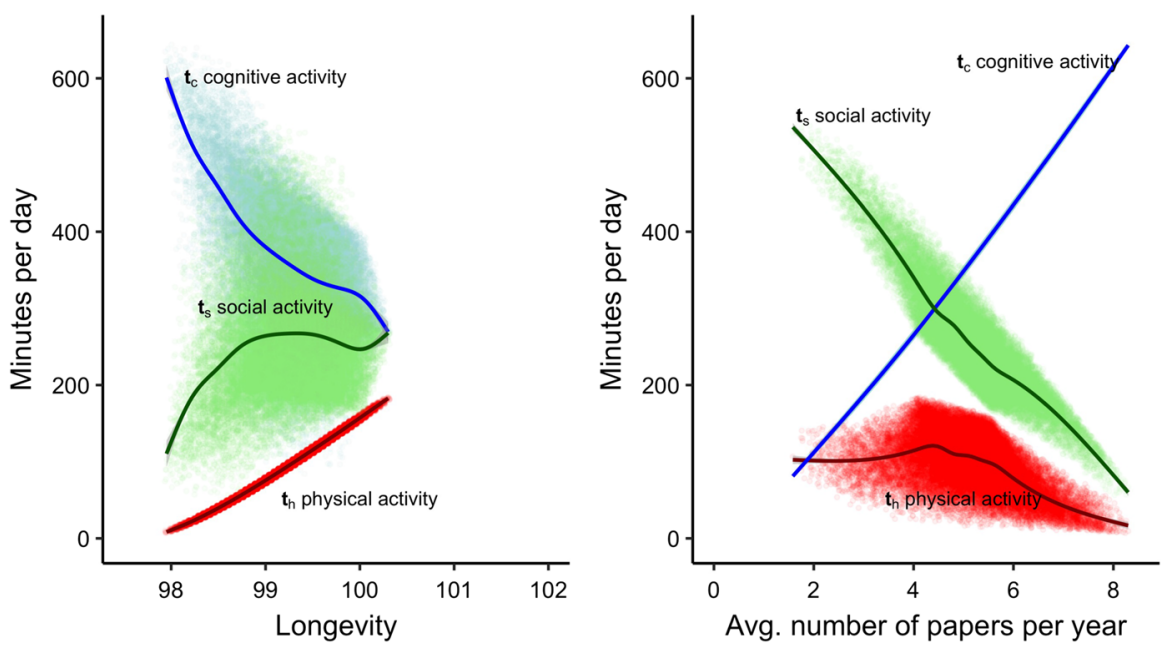

Fig. 8 Average time devoted to each activity over the remaining life by longevity (left panel) and average number of papers per year (right panel): health dependent utility model. Note: Solid lines represent the conditional mean over the average time devoted to each activity

Fig. 8 shows that the distribution of time across the different activities conditional on longevity and average number of papers per year does not significantly differ from Fig. 3.

Table 5 reports the descriptive statistics of the preference parameters characterizing cases A, B, and C in the "health dependent utility" model. The main difference between Tables 2 and 5 is in the salvage parameter. In particular, the simulation results show that in order to obtain similar values of longevity and average number of papers, the scholar in the health dependent utility model values less the stock of knowledge left 
Table 5 Descriptive statistics of the preference parameters for cases A, B, and C

\begin{tabular}{|c|c|c|c|c|c|c|c|}
\hline & \multirow[t]{2}{*}{ Symbol } & \multicolumn{2}{|l|}{ Case A } & \multicolumn{2}{|l|}{ Case B } & \multicolumn{2}{|l|}{ Case $\mathrm{C}$} \\
\hline & & Mean & $\mathrm{SD}$ & Mean & SD & Mean & SD \\
\hline Avg. number of papers per year & $p$ & 5.01 & 0.15 & 4.99 & 0.14 & 4.07 & 0.13 \\
\hline Longevity & $\alpha$ & 98.62 & 0.12 & 99.92 & 0.10 & 99.98 & 0.13 \\
\hline Weight cognitive activity & $\phi_{c}$ & 5.342 & 1.422 & 17.092 & 2.228 & 15.245 & 2.662 \\
\hline Weight social activity & $\phi_{s}$ & 11.937 & 4.006 & 18.720 & 1.261 & 18.517 & 1.280 \\
\hline Subjective discount factor & $\rho$ & 0.016 & 0.004 & 0.003 & 0.002 & 0.004 & 0.003 \\
\hline Salvage parameter & $\kappa$ & 1.820 & 1.125 & 2.492 & 0.584 & 0.492 & 0.422 \\
\hline
\end{tabular}

to future generations. This is because the strength of the utility loss of not enjoying the "glory" from the stock of knowledge left to future generations $(\kappa)$ on the shadow price of health deficits $\left(-\lambda_{D}(\alpha)\right)$ is higher in the health dependent utility model. See Eqs. (33) and (33ii) in "Appendix B". As a consequence, it is necessary a lower value of $\kappa$ to reach the same longevity as in the benchmark model.

\subsection{Cognitive decay}

As a follow-up of the previous model, we now study the case in which an increase in health deficits affects negatively on the efficiency of cognitive activities. This alternative model can be implemented by assuming that the change in time of papers published is reduced by the factor $(\mathcal{D} / D)^{v}$. As a result, the number of papers published at time $t$ is given by

$$
\dot{K}=\theta(\mathcal{D} / D)^{\sigma} \mathrm{t}_{c}^{\gamma} K^{\beta}
$$

where $\sigma \in(0,1)$ accounts for the rate of decline in the efficiency of the cognitive activity due to a deterioration of the health status. Equation (22) implies that the number of paper published depends on two counteracting forces. On the one side we have that the productivity of an hour worked on cognitive activities increases because of the experience accumulated writing papers; i.e. $K^{\beta}$. Whereas, on the other side, we have that the productivity of an hour devoted to cognitive activities decreases as the number of health deficits rises; i.e. $(\mathcal{D} / D)^{\sigma}$. Following a similar strategy as in the previous model, we set $\mathcal{D}$ at the value of the health deficit index at age 78 and $\sigma$ at 0.5 . Therefore, we consider that the efficiency of the time devoted to cognitive activities declines by $25 \%$ at the end of life, ceteris paribus the remaining variables.

Figure 9 shows the combination of possible values of the average number of papers published per year and longevity under the benchmark model and the "cognitive decay" model. Red circles depict the result for the benchmark model, while blue triangles depict the results for the "cognitive decay" model. As we can see in Fig. 9 the "cognitive decay" model gives very similar longevity values compared to the benchmark model (see the marginal distribution on the right margin), but lower average number of papers per year. This is due to the assumption that the efficiency of cognitive activity declines as our scholar ages. 


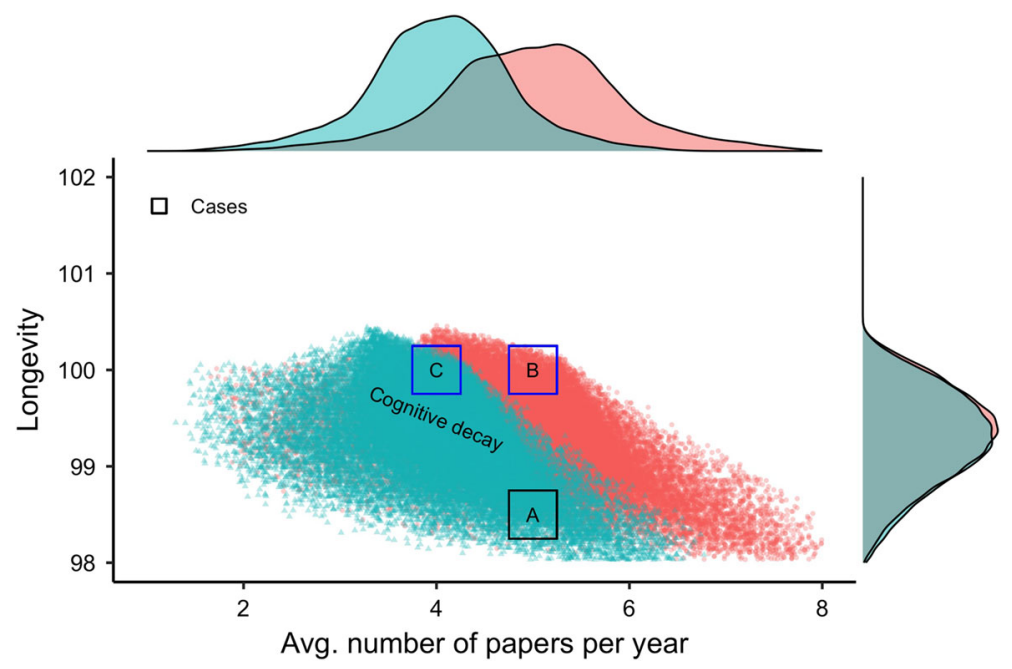

Fig. 9 Set of possible longevity $(\alpha)$ and average number of papers $(p)$ generated with the benchmark model (red circles) and the cognitive decay model (blue triangles) (color figure online)
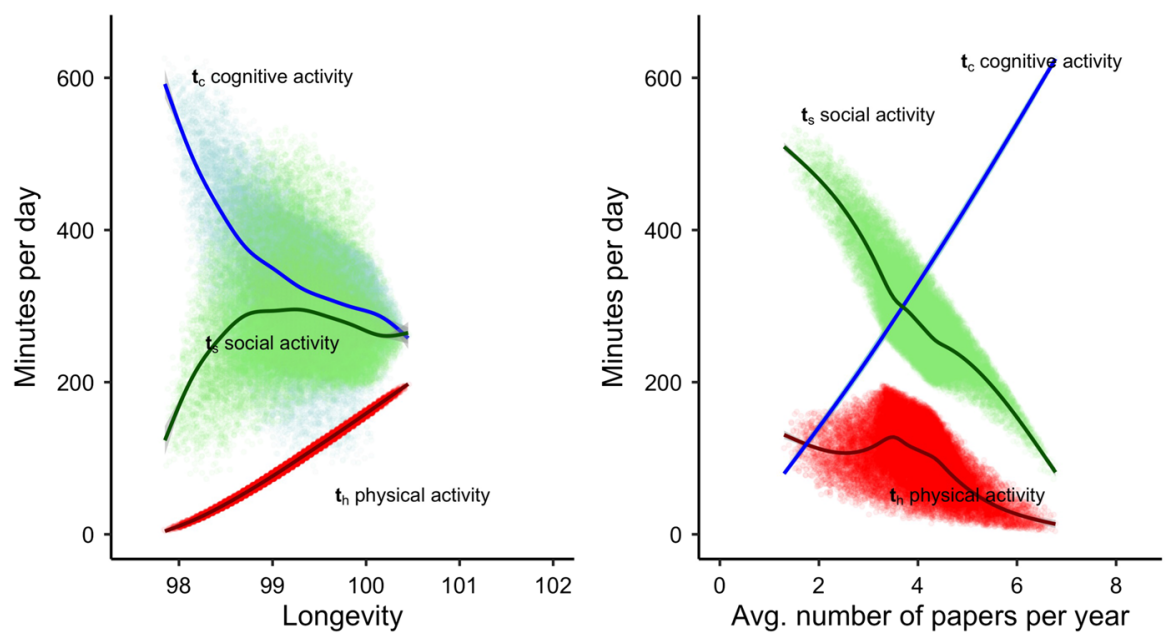

Fig. 10 Average time devoted to each activity over the remaining life by longevity (left panel) and average number of papers per year (right panel): cognitive decay model. Note: Solid lines represent the conditional mean over the average time devoted to each activity

The average time devoted to each activity over the remaining life in the cognitive decay model is shown in Fig. 10. Comparing Figs. 3 and 10 we can observe that the average time spent on cognitive activities, conditional on the average number of papers per year, increases in the cognitive decay model relative to the benchmark model. However, there is no significant change between the benchmark model and the cognitive decay model on the time spent on the different activities in order to reach a specific longevity. Therefore, despite the fact that individuals devote the same average 
Table 6 Descriptive statistics of the preference parameters for cases A, B, and C

\begin{tabular}{|c|c|c|c|c|c|c|c|}
\hline & \multirow[t]{2}{*}{ Symbol } & \multicolumn{2}{|l|}{ Case A } & \multicolumn{2}{|c|}{ Case B } & \multicolumn{2}{|l|}{ Case C } \\
\hline & & Mean & SD & Mean & SD & Mean & SD \\
\hline Avg. number of papers per year & $p$ & 5.00 & 0.14 & - & - & 3.98 & 0.14 \\
\hline Longevity & $\alpha$ & 98.57 & 0.13 & - & - & 99.94 & 0.11 \\
\hline Weight cognitive activity & $\phi_{c}$ & 7.992 & 2.966 & - & - & 16.885 & 2.233 \\
\hline Weight social activity & $\phi_{S}$ & 11.376 & 2.696 & - & - & 18.513 & 1.471 \\
\hline Subjective discount factor & $\rho$ & 0.014 & 0.004 & - & - & 0.003 & 0.002 \\
\hline Salvage parameter & $\kappa$ & 3.532 & 1.028 & - & - & 2.800 & 0.862 \\
\hline
\end{tabular}

time across the different activities, in the cognitive decay model our scholar will reach the same longevity but lower average number of papers.

Table 6 shows that publishing an average of five papers per year and reaching a longevity of one hundred years (case B) is not possible in this model. From cases A and $\mathrm{C}$ we can see that in order to reach the same number of papers as in the benchmark model, scholars should give in the cognitive decay model a higher preference towards cognitive activities and value more the knowledge left to future generations than in the benchmark model.

\section{Conclusions}

As the life phase spent in retirement has increased over time, activity patterns in retirement have changed as well. Recent studies (e.g. Mergenthaler et al. 2018) have investigated the variation in these activity patterns identifying various types of retirees. However, so far, the role of an active retirement and its impact on the retiree's health (and hence life expectancy) has not formally been investigated.

In this paper we built up a model of optimal time allocation in retirement allowing for feedback mechanisms of the choice of activity on the agent's health. We calibrate our model to the life of a scientific scholar and assume that time in retirement can be allocated between cognitive activities, social activities and physical activity. While cognitive activity increases the stock of scientific papers produced, physical activity increases health and hence the life span of the retiree. We assume that our scholar derives utility out of cognitive and social activities and disutility from physical activity. Moreover, we introduce a salvage value for the papers written until the end of life.

Depending on the time preference of the retiree, the valuation of the salvage value of papers written, and the utility weight on the various activities we first study the optimal allocation of time in retirement and its impact on longevity and on the number of papers written. Then, we use the numerical results to investigate the behavioral characteristics that support specific outcomes of longevity and number of papers as well as the optimal allocation of time to reach these specific outcomes. Overall our results show a positive correlation between living longer and the joy of producing papers, being more patient, and the utility from leaving a large stock of knowledge to future generations. 
To further study how physical activity impacts on longevity we also analyze three extensions of our benchmark model. In the first extension we have assumed that our scholar derives no disutility from physical activity. Such a set up allows for a pronounced shift in the longevity as the optimal time spent on physical activity increases, but at the expense of less papers being written. Since the health status will not only determine the longevity but may also affect cognitive and social outcomes, we have introduced two additional alternative specifications of our model framework. A model in which health is multiplicatively linked to the utility gained out of social and cognitive activities and another model in which the efficiency of cognitive activities to produce papers is reduced if health deteriorates. Our results indicate that these indirect effects of the health status on utility (case a) and on the efficiency of producing papers (case b) imply that in order to obtain the same value of the longevity as in the benchmark case the valuation of the stock of knowledge left to future generations needs to be reduced (case a) and alternatively increased (case b).

The results obtained in this paper assume that the time spent on each activity is mutually exclusive. Further possible extensions of the model set up can allow for the fact that either the time spent on physical activity can contribute to the time spent on social activities (e.g. exercising together with friends), or the time spent on physical activity can contribute to the time spent on cognitive activities (e.g. gaining additional innovative ideas during physical activity). Alternatively, we may also allow for interactions of the time spent on one activity to increase the efficiency of the time spent on other activities. Thus, our model should be considered a first attempt to gain more insight into the mutual feedback between an active retirement and longevity.

Acknowledgements Open access funding provided by TU Wien (TUW). We like to thank Mrs. Rivic for her research assistance and Gustav Feichtinger, Dieter Grass, Vladimir Veliov, Stefan Wrzaczek and participants at the symposium on occasion of the award of the Wissenschaftpreis der Österreichischen Forschungsgemeinschaft for useful comments and suggestions.

Open Access This article is distributed under the terms of the Creative Commons Attribution 4.0 International License (http://creativecommons.org/licenses/by/4.0/), which permits unrestricted use, distribution, and reproduction in any medium, provided you give appropriate credit to the original author(s) and the source, provide a link to the Creative Commons license, and indicate if changes were made.

\section{A Appendix: The maximum principle/optimality conditions}

We use Portryagin's Maximum Principle to derive the necessary conditions for an optimal solution of a problem with variable (free) terminal time and state constraints (Grass et al. 2008). Assuming a static nonlinear optimization, the Lagrangian associated to problem (7) is

$$
\begin{aligned}
\mathcal{L}(\alpha)= & \int_{x}^{\alpha} e^{-\rho(t-x)} U(\cdot) d t+\int_{x}^{\alpha} e^{-\rho(t-x)} \lambda_{K}(t)\left(\theta \mathrm{t}_{c}(t)^{\gamma} K(t)^{\beta}-\dot{K}(t)\right) d t \\
& +\int_{x}^{\alpha} e^{-\rho(t-x)} \lambda_{D}(t)\left(\mu\left(D(t)-A \mathrm{t}_{h}(t)^{\eta}-a\right)-\dot{D}(t)\right) d t \\
& +e^{-\rho(\alpha-x)} S(K(\alpha))+v(\bar{D}-D(\alpha)) .
\end{aligned}
$$


where $v>0$ is the Kuhn-Tucker multiplier associated to the maximum number of deficits. We obtain the following necessary conditions for a maximum of (23) for all $t \in[x, \alpha]$ :

$$
\begin{gathered}
\frac{\partial \mathcal{H}}{\partial \mathrm{t}_{i}}=0 \quad \text { for } i \in\{c, h\} \\
\dot{\lambda}_{K}-\rho \lambda_{K}=-\frac{\partial \mathcal{H}}{\partial K}, \\
\dot{\lambda}_{D}-\rho \lambda_{D}=-\frac{\partial \mathcal{H}}{\partial D}, \\
\lambda_{K}\left(\alpha^{*}\right)=\frac{\partial S}{\partial K}>0, \\
\lambda_{D}\left(\alpha^{*}\right)=-v<0, \\
\mathcal{H}\left(\mathrm{t}_{i}^{*}\left(\alpha^{*}\right), K^{*}\left(\alpha^{*}\right), D^{*}\left(\alpha^{*}\right), \lambda_{K}\left(\alpha^{*}\right), \lambda_{D}\left(\alpha^{*}\right), \alpha^{*}\right)=\rho S\left(K^{*}\left(\alpha^{*}\right)\right)-\left.\frac{d S\left(K^{*}(\alpha)\right)}{d \alpha}\right|_{\alpha=\alpha^{*}}, \\
D(24 \mathrm{f})
\end{gathered}
$$

where $\mathcal{H}$ is the Hamiltonian associated to each particular problem. Following Feichtinger and Hartl (1986, see p. 116) and Cerdá (2001, see pp. 208-211), Eq. (24f) is a necessary condition for (23) to achieve its maximum at $\alpha^{*}$ (i.e. $d \mathcal{L}\left(\alpha^{*}\right) / d \alpha=0$ ).

\section{B Appendix: Solutions}

Given (24a)-(24g) in this section we derive the optimal conditions for each model implemented by using the Portryagin's Maximum Principle. In all of them we assume that the salvage-value function is $S(K(\alpha))=\kappa K(\alpha)$.

\section{B.1 Benchmark model}

The current-value Hamiltonian associated to the benchmark model is

$$
\mathcal{H}=\phi_{s} \log \mathrm{t}_{s}+\phi_{c} \log \mathrm{t}_{c}-\phi_{h} \frac{\mathrm{t}_{h}^{1+\omega}}{1+\omega}+\lambda_{K} \theta \mathrm{t}_{c}^{\gamma} K^{\beta}+\lambda_{D} \mu\left(D-A \mathrm{t}_{h}^{\eta}-a\right),
$$

where $\lambda_{K}$ and $\lambda_{D}$ are the adjoint variables associated to the stock of knowledge and the stock of health, respectively. From (24a)-(24g) and (25) we have that the first-order conditions for the optimal time devoted to cognitive, physical, and social activities are: 


$$
\begin{array}{r}
\frac{\phi_{s}}{\mathrm{t}_{s}}-\frac{\phi_{c}}{\mathrm{t}_{c}}=\frac{\gamma}{\mathrm{t}_{c}} \lambda_{K} \theta \mathrm{t}_{c}^{\gamma} K^{\beta}, \\
\frac{\phi_{s}}{\mathrm{t}_{s}}+\phi_{h} \mathrm{t}_{h}{ }^{\omega}=\frac{\eta}{\mathrm{t}_{h}}\left(-\lambda_{D}\right) \mu A \mathrm{t}_{h}^{\eta}, \\
\mathrm{t}_{s}=T-\mathrm{t}_{c}-\mathrm{t}_{h} .
\end{array}
$$

The envelope conditions, given by,

$$
\begin{array}{r}
\dot{\lambda}_{K}=\lambda_{K}\left(\rho-\beta \frac{\dot{K}}{K}\right), \\
\dot{\lambda}_{D}=\lambda_{D}(\rho-\mu),
\end{array}
$$

satisfy the transversality conditions: $\lambda_{K}(\alpha)=\kappa$ and $\lambda_{D}(\alpha)=-v$. Moreover, from (24) we have that the optimal terminal age condition satisfies the following two equations

$$
\begin{aligned}
D\left(\alpha^{*}\right)= & \bar{D}, \\
-\lambda_{D}\left(\alpha^{*}\right)= & \frac{\phi_{s} \log \mathrm{t}_{s}\left(\alpha^{*}\right)+\phi_{c} \log \mathrm{t}_{c}\left(\alpha^{*}\right)-\phi_{h} \frac{\mathrm{t}_{h}\left(\alpha^{*}\right)^{1+\omega}}{1+\omega}}{\mu\left(D\left(\alpha^{*}\right)-a\right)} \\
& +\frac{\lambda_{K}\left(\alpha^{*}\right) \theta \mathrm{t}_{c}\left(\alpha^{*}\right)^{\gamma} K\left(\alpha^{*}\right)^{\beta}+\kappa \dot{K}\left(\alpha^{*}\right)-\lambda_{D}\left(\alpha^{*}\right) \mu A \mathrm{t}_{h}\left(\alpha^{*}\right)^{\eta}-\rho \kappa K^{*}\left(\alpha^{*}\right)}{\mu\left(D\left(\alpha^{*}\right)-a\right)} .
\end{aligned}
$$

Using (26), (27), and (31) in (32) we obtain

$$
\begin{aligned}
-\lambda_{D}\left(\alpha^{*}\right)= & \frac{\phi_{s} \log \mathrm{t}_{s}\left(\alpha^{*}\right)+\phi_{c} \log \mathrm{t}_{c}\left(\alpha^{*}\right)+\left(\frac{1}{\eta}-\frac{1}{1+\omega}\right) \phi_{h} \mathrm{t}_{h}\left(\alpha^{*}\right)^{1+\omega}}{\mu(\bar{D}-a)} \\
& +\frac{2 \frac{\mathrm{t}_{c}\left(\alpha^{*}\right)}{\gamma}\left(\frac{\phi_{s}}{\mathrm{t}_{s}\left(\alpha^{*}\right)}-\frac{\phi_{c}}{\mathrm{t}_{c}\left(\alpha^{*}\right)}\right)+\frac{\phi_{s}}{\eta} \frac{\mathrm{t}_{h}\left(\alpha^{*}\right)}{\mathrm{t}_{s}\left(\alpha^{*}\right)}-\rho \kappa K^{*}\left(\alpha^{*}\right)}{\mu(\bar{D}-a)},
\end{aligned}
$$

which is equivalent to Eq. (18).

\section{B.2 Sportsman}

The Hamiltonian associated to the "sportsman" model is

$$
\mathcal{H}=\phi_{s} \log \mathrm{t}_{s}+\phi_{c} \log \mathrm{t}_{c}+\lambda_{K} \theta \mathrm{t}_{c}^{\gamma} K^{\beta}+\lambda_{D} \mu\left(D-A \mathrm{t}_{h}^{\eta}-a\right)
$$

By assuming that $\phi_{h}=0$ the first-order condition of the optimal time devoted to physical activity and the terminal age condition change with respect to the benchmark model. In particular, we have that the marginal utility cost of the time devoted to physical activity diminishes 


$$
\frac{\phi_{s}}{\mathrm{t}_{s}}=\frac{\eta}{\mathrm{t}_{h}} \lambda_{D} \mu A \mathrm{t}_{h}^{\eta}
$$

as well as that the shadow price of avoiding the accumulation of health deficits

$$
\begin{aligned}
-\lambda_{D}\left(\alpha^{*}\right)= & \frac{\phi_{s} \log \mathrm{t}_{s}\left(\alpha^{*}\right)+\phi_{c} \log \mathrm{t}_{c}\left(\alpha^{*}\right)}{\mu\left(D\left(\alpha^{*}\right)-a\right)} \\
& +\frac{2 \frac{\mathrm{t}_{c}\left(\alpha^{*}\right)}{\gamma}\left(\frac{\phi_{s}}{\mathrm{t}_{s}\left(\alpha^{*}\right)}-\frac{\phi_{c}}{\mathrm{t}_{c}\left(\alpha^{*}\right)}\right)+\frac{\phi_{s}}{\eta} \frac{\mathrm{t}_{h}\left(\alpha^{*}\right)}{\mathrm{t}_{s}\left(\alpha^{*}\right)}-\rho \kappa K^{*}\left(\alpha^{*}\right)}{\mu(\bar{D}-a)} .
\end{aligned}
$$

As a consequence, given the optimal time devoted to cognitive activities, those individuals, who behaves as a 'sportsman', spend more time on physical activity.

\section{B.3 Health dependent utility}

The Hamiltonian associated to the "health dependent utility" model is

$$
\mathcal{H}=\left(\frac{\mathcal{D}}{D}\right)^{\varepsilon}\left(\phi_{s} \log \mathrm{t}_{s}+\phi_{c} \log \mathrm{t}_{c}\right)-\phi_{h} \frac{\mathrm{t}_{h}^{1+\omega}}{1+\omega}+\lambda_{K} \theta \mathrm{t}_{c}^{\gamma} K^{\beta}+\lambda_{D} \mu\left(D-A \mathrm{t}_{h}^{\eta}-a\right),
$$

where $\mathcal{D} \in(0, D)$ is an arbitrary constant value and $\varepsilon>0$ accounts for the rate of decline in utility caused by increases in health deficits. By assuming that the utility from cognitive and social activities depend negatively on the health status, there is a change not only in the first-order conditions for cognitive and physical activities, but also in the shadow price of health and the terminal age conditions with respect to the benchmark model. Thus, we have that, ceteris paribus, the marginal utility costs of cognitive and physical activities in the "health dependent utility" model decline compared to the benchmark model

$$
\begin{aligned}
& \left(\frac{\mathcal{D}}{D}\right)^{\varepsilon}\left(\frac{\phi_{s}}{\mathrm{t}_{s}}-\frac{\phi_{c}}{\mathrm{t}_{c}}\right)=\frac{\gamma}{\mathrm{t}_{c}} \lambda_{K} \theta \mathrm{t}_{c}^{\gamma} K^{\beta}, \\
& \left(\frac{\mathcal{D}}{D}\right)^{\varepsilon} \frac{\phi_{s}}{\mathrm{t}_{s}}+\phi_{h} \mathrm{t}_{h}{ }^{\omega}=\frac{\eta}{\mathrm{t}_{h}} \lambda_{D} \mu A \mathrm{t}_{h}^{\eta} .
\end{aligned}
$$

The shadow price of avoiding the accumulation of health deficits at the end of life decreases relative to that in the benchmark model 


$$
\begin{aligned}
-\lambda_{D}\left(\alpha^{*}\right)= & \frac{\left(\frac{\mathcal{D}}{D}\right)^{\varepsilon}\left(\phi_{s} \log \mathrm{t}_{s}\left(\alpha^{*}\right)+\phi_{c} \log \mathrm{t}_{c}\left(\alpha^{*}\right)\right)+\left(\frac{1}{\eta}-\frac{1}{1+\omega}\right) \phi_{h} \mathrm{t}_{h}\left(\alpha^{*}\right)^{1+\omega}}{\mu(\bar{D}-a)} \\
& +\frac{\left(\frac{\mathcal{D}}{D}\right)^{\varepsilon}\left(2 \frac{\mathrm{t}_{c}\left(\alpha^{*}\right)}{\gamma}\left(\frac{\phi_{s}}{\mathrm{t}_{s}\left(\alpha^{*}\right)}-\frac{\phi_{c}}{\mathrm{t}_{c}\left(\alpha^{*}\right)}\right)+\frac{\phi_{s}}{\eta} \frac{\mathrm{t}_{h}\left(\alpha^{*}\right)}{\mathrm{t}_{s}\left(\alpha^{*}\right)}\right)-\rho \kappa K^{*}\left(\alpha^{*}\right)}{\mu(\bar{D}-a)} .
\end{aligned}
$$

But the rate of decline with age of the price of health deficits is slowed down.

$$
\dot{\lambda}_{D}=\lambda_{D}(\rho-\mu)+\frac{\varepsilon}{D}\left(\frac{\mathcal{D}}{D}\right)^{\varepsilon}\left(\phi_{s} \log \mathrm{t}_{s}+\phi_{c} \log \mathrm{t}_{c}\right) .
$$

Therefore, individuals, who behave as in the "health dependent utility" model, spend at the end of life less time on physical activities than those in the benchmark model, but it is more likely that they spend more time on physical activity early in life.

\section{B.4 Cognitive decay}

The Hamiltonian associated to the "cognitive decay" model is

$$
\mathcal{H}=\phi_{s} \log \mathrm{t}_{s}+\phi_{c} \log \mathrm{t}_{c}-\phi_{h} \frac{\mathrm{t}_{h}^{1+\omega}}{1+\omega}+\lambda_{K} \theta\left(\frac{\mathcal{D}}{D}\right)^{\sigma} \mathrm{t}_{c}^{\gamma} K^{\beta}+\lambda_{D} \mu\left(D-A \mathrm{t}_{h}^{\eta}-a\right),
$$

where $\sigma>0$ represents the rate of decline in the production of papers caused by increases in health deficits.

Under the "cognitive decay" model only the first-order condition for the optimal time devoted to cognitive activities changes with respect to the benchmark model. The new first-order condition for $t_{c}$ becomes

$$
\frac{\phi_{s}}{\mathrm{t}_{s}}-\frac{\phi_{c}}{\mathrm{t}_{c}}=\frac{\gamma}{\mathrm{t}_{c}} \lambda_{K} \theta\left(\frac{\mathcal{D}}{D}\right)^{\sigma} \mathrm{t}_{c}^{\gamma} K^{\beta},
$$

which implies that the cognitive activity in the "cognitive decay" model must be lower than that in the benchmark model.

\section{References}

Cerdá E (2001) Optimización Dinámica. Prentice Hall, Madrid

Dalgaard CJ, Strulik H (2014) Optimal aging and death: understanding the Preston curve. J Eur Econ Assoc 12(3):672-701

Dalgaard CJ, Strulik H (2017) The genesis of the golden age: accounting for the rise in health and leisure. Rev Econ Dyn 24:132-151

Feichtinger G, Hartl RF (1986) Optimale Kontrolle Ökonomischer Prozesse: Anwendungen des Maximumprinzips in den Wirtschaftswissenschaften. Walter de Gruyter, Berlin 
Feichtinger G, Grass D, Kort PM (2018) Optimal scientific production over the life cycle. J Econ Dyn Control 108:103752

Grass D, Caulkins JP, Feichtinger G, Tragler G, Behrens DA (2008) Optimal control of nonlinear processes: with applications in drugs, corruption, and terror. Springer, Berlin

Human Mortality Database (2018) University of California, Berkeley (USA), and Max Planck Institute for Demographic Research (Germany). www.mortality.org (data downloaded on 3 Sept 2018)

Mergenthaler A, Sackreuther I, Staudinger UM (2018) Productive activity patterns among 60-70-year-old retirees in Germany. Ageing Soc 39:1-30

Mitnitski AB, Mogilner AJ, MacKnight C, Rockwood K (2002a) The accumulation of deficits with age and possible invariants of aging. Sci World J 2:1816-1822

Mitnitski AB, Mogilner AJ, MacKnight C, Rockwood K (2002b) The mortality rate as a function of accumulated deficits in a frailty index. Mech Ageing Dev 123(11):1457-1460

Schuenemann J, Strulik H, Trimborn T (2017) Going from bad to worse: adaptation to poor health, health spending, longevity, and the value of life. J Econ Behav Organ 140:130-146

Sprod J, Olds T, Brown W, Burton N, van Uffelen J, Ferrar K, Maher C (2017) Changes in use of time across retirement: a longitudinal study. Maturitas 100:70-76

Strulik H (2018) The return to education in terms of wealth and health. J Econ Ageing 12:1-14

Strulik H (2019) An economic theory of depression and its impact on health behavior and longevity. J Econ Behav Organ 158:269-87

Winkler-Dworak M (2008) The low mortality of a learned society. Eur J Population 24:405-424

Winkler-Dworak M, Kaden H (2014) The longevity of academicians: evidence from the Saxonian Academy of Sciences and Humanities in Leipzig. Vienna Yearb Popul Res 11:185-204

Publisher's Note Springer Nature remains neutral with regard to jurisdictional claims in published maps and institutional affiliations. 